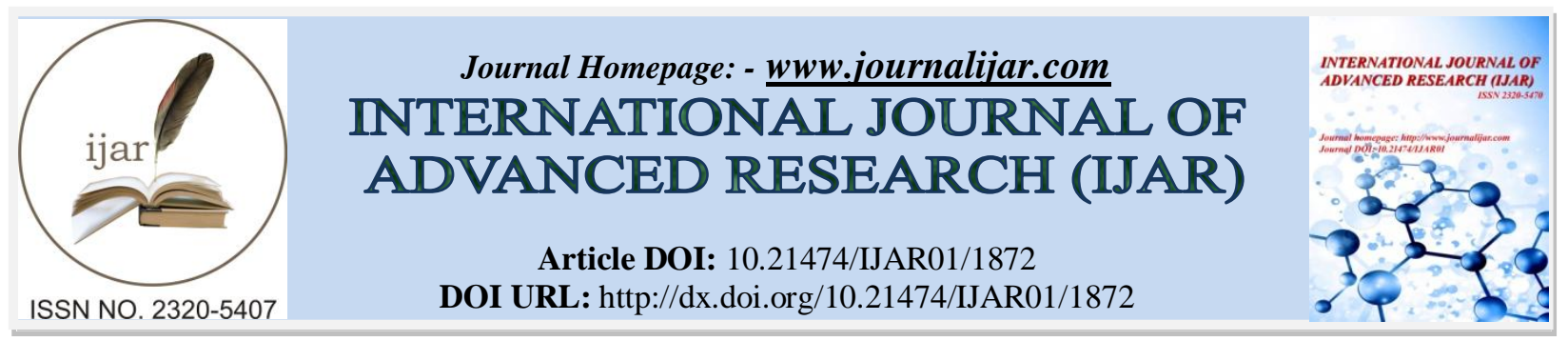

RESEARCH ARTICLE

\title{
THE AGING BRAIN: NANOTECHNOLOGY SOLUTIONS WITH DIAGNOSTIC METHODS AND RECENT ADVANCES.
}

\section{Ayesha Habeeb ${ }^{*}$ and Amtun Noor.}

Deccan school of pharmacy, Department of pharmaceutics ,Dar us salam, Aghapura, Nampally, Hyderabad Telangana, India.

\section{Manuscript Info}

Manuscript History

Received: 12 August 2016

Final Accepted: 14 September 2016

Published: October 2016

Key words:-

Alzheimer's disease, Nanotechnology, Blood brain barrier(BBB)

\section{Abstract}

Alzheimer's disease (AD) is the leading cause of dementia is a devastating degenerative disease of brain that results in progressive cognitive impairment and functional disability. AD lacks definite diagnostic approaches and effective cure at the present. Moreover, the currently available diagnostic tools are not sufficient for an early screening of AD in order to start preventive approaches. Recently the emerging field of nanotechnology has promised new techniques to solve some of the $\mathrm{AD}$ challenges. In this review, we present the promises that nanotechnology brings in research on the AD diagnosis ,Proposed invitro and invivo nanodiagnostic approaches, Nanoformulations investigated and therapy through nanotechnology. Applications of nanotechnology in AD therapy including neuroprotections against oxidative stress and anti-amyloid therapeutics, neuroregeneration and drug delivery beyond the blood brain barrier (BBB) are discussed and analyzed. All these applications could improve the treatment approach of AD and other neurodegenerative diseases. The complete cure of $\mathrm{AD}$ may become feasible by a combination of nanotechnology and some other novel approaches, like stem cell technology.

Copy Right, IJAR, 2016,. All rights reserved.

\section{Introduction:-}

Alzheimer's disease (AD) is an acquired disorder of cognitive and behavioral impairment that is an incurable disease with a long and progressive course. In $\mathrm{AD}$, plaques develop in the hippocampus, a structure deep in the brain that helps to encode memories, and in other areas of the cerebral cortex that are used in thinking and decision making. Alois Alzheimer defined "senile dementia" more than a century ago with a remarkable accuracy" ${ }^{1,2}$ It is now known as the Alzheimer's Disease (AD), and is the main cause of the dementia syndrome ${ }^{3}$. The incidence and prevalence of AD increase with age. Since the elderly population is growing worldwide, AD is quickly becoming one of the major universal healthcare problems ${ }^{4}$. Today, however, there are neither precise diagnostic approaches nor effective therapeutic agents available for Alzheimer's disease. The degeneration of nervous tissue begins many years to even decades before the patient experiences any of Alzheimer's disease symptoms ${ }^{5,7}$. On the other hand, the currently available therapeutics for AD, only act to lower its symptoms ${ }^{8}$. Therefore, whether the disease is diagnosed early enough or not, the conventional medical approaches are incapable of complete cessation or reversal of the disease progress. The uncovering potentials of nanotechnology have been opening new chapters in many aspects of our lives, specially diagnosis and treatment of human diseases ${ }^{9,11}$. Through nanotechnology, the controllable

Corresponding Author:- Ayesha Habeeb.

Address:- Deccan school of pharmacy, Department of pharmaceutics,dar us salam, aghapura, nampally, hyderabad telangana, India. 
production of desired structures and devices with, at least, one dimension in nanoscale (1- $100 \mathrm{~nm})$ is presently achievable ${ }^{12,13}$. Nanotechnology is advancing molecular detection, drug discovery, delivery and monitoring for a number of ever-challenging human diseases, including cancer and neurodegenerative disorders ${ }^{10,14}$. The success of nanotechnology approaches towards diagnosis and treatment of AD presented in this paper, demonstrates the role of interdisciplinary research for the early diagnosis and possible cure for AD.

\section{Structure of blood brain barrier (bbb) and transport mechanism:-}

The $\mathrm{BBB}$ is considered to be a dynamic and complex barrier separating blood and the central nervous system that strictly controls the exchanges between the compartments of blood and brain ${ }^{15}$. Blood brain barrier is a natural biological barrier that plays a crucial role in the protection of the brain by restricting the entry of untoward substances such as toxic molecules, pathogens, and numerous other external molecules and thereby maintaining the brain homeostasis. An illustration of blood brain barrier is shown in Fig.1. The endothelial cells present in brain differ significantly from cells present in other parts of the body, due to the presence of intracellular tight junctions, lesser paracellular diffusion of hydrophilic molecules, presence of relatively high number of mitochondrial cells and thereby with high metabolic activity, and a relatively higher number of active transporters ${ }^{16}$.The basal lamina, composed mainly of collagen, glycoproteins, and proteoglycans, is involved in the dynamic regulation of blood brain barrier with the aid of multiple basal lamina proteins, matrix metalloproteases, their inhibitors, and the tissue inhibitors of metalloproteases. Brain microvessels have numerous pericytes and ratio of pericytes to endothelial cells was linked with the barrier capacity. Endothelium, pericytes, perivascular astrocytes are very close contact with neuronal projections.

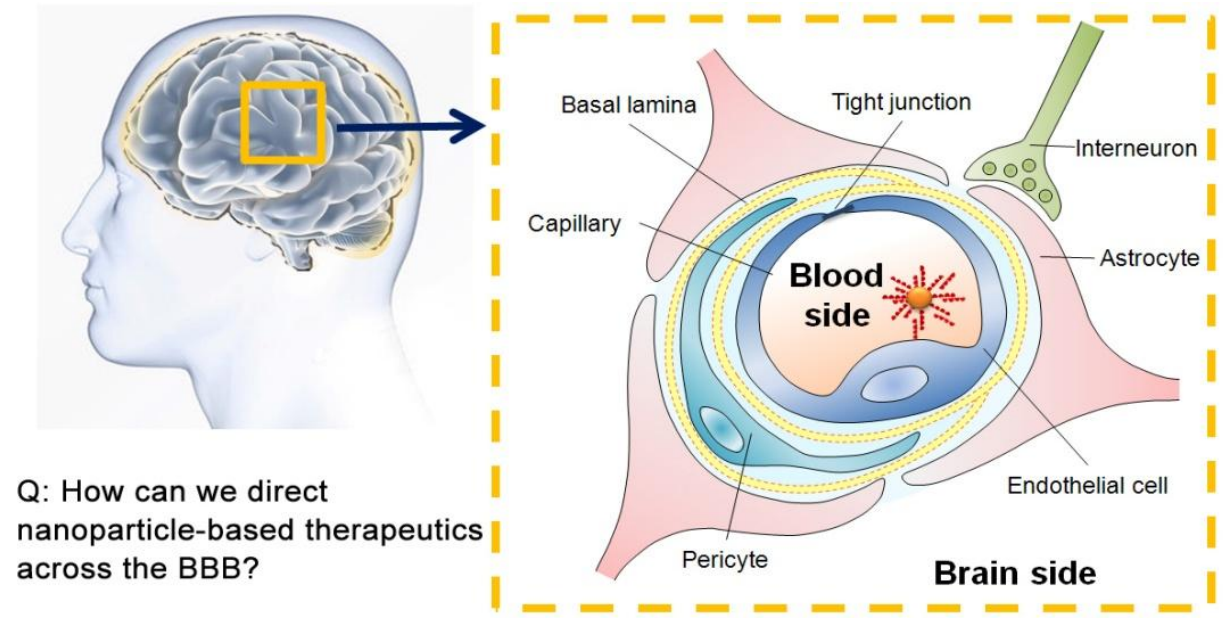

Figure 1:- Schematic representation of blood brain barrier (BBB)

\section{General transport mechanisms across bbb:-}

The major transport mechanisms to the BBB are depicted in Fig. 2. These include paracellular aqueous pathway, transcellular lipophilic pathway, transport protein pathway, receptor mediated transcytosis, and adsorptive $\operatorname{transcytosis}^{17}$. Paracellular aqueous pathway is a rare pathway across BBB through which small water-soluble molecules diffuse into the brain. Lipophilic molecules such as alcohol, steroid hormones etc.penetrate transcellularly by dissolving in their lipid plasma membrane. In carrier-mediated transport, a protein transporter binds to glucose or amino acids which triggers a conformational change in the protein and helps in the transport of the molecule to the other side. The receptor-mediated transcytosis type of transport mechanism is for the selective uptake of macromolecules. These systems include receptors for transferrin, insulin, lipoprotein etc and those are also explored for the ligand based nanoformulations, which have been discussed in detail in later sections of this review. Another kind of transport mechanism, called adsorptive-mediated transcytosis is triggered by an electrostatic interaction between a positively charged substance such as charged moiety of a peptide, and the negatively charged plasma membrane surface such as heparin sulphate proteoglycans. The components of BBB and specific transport mechanisms make BBB highly dynamic and complex, thereby restricting the entry of various drug molecules.. 


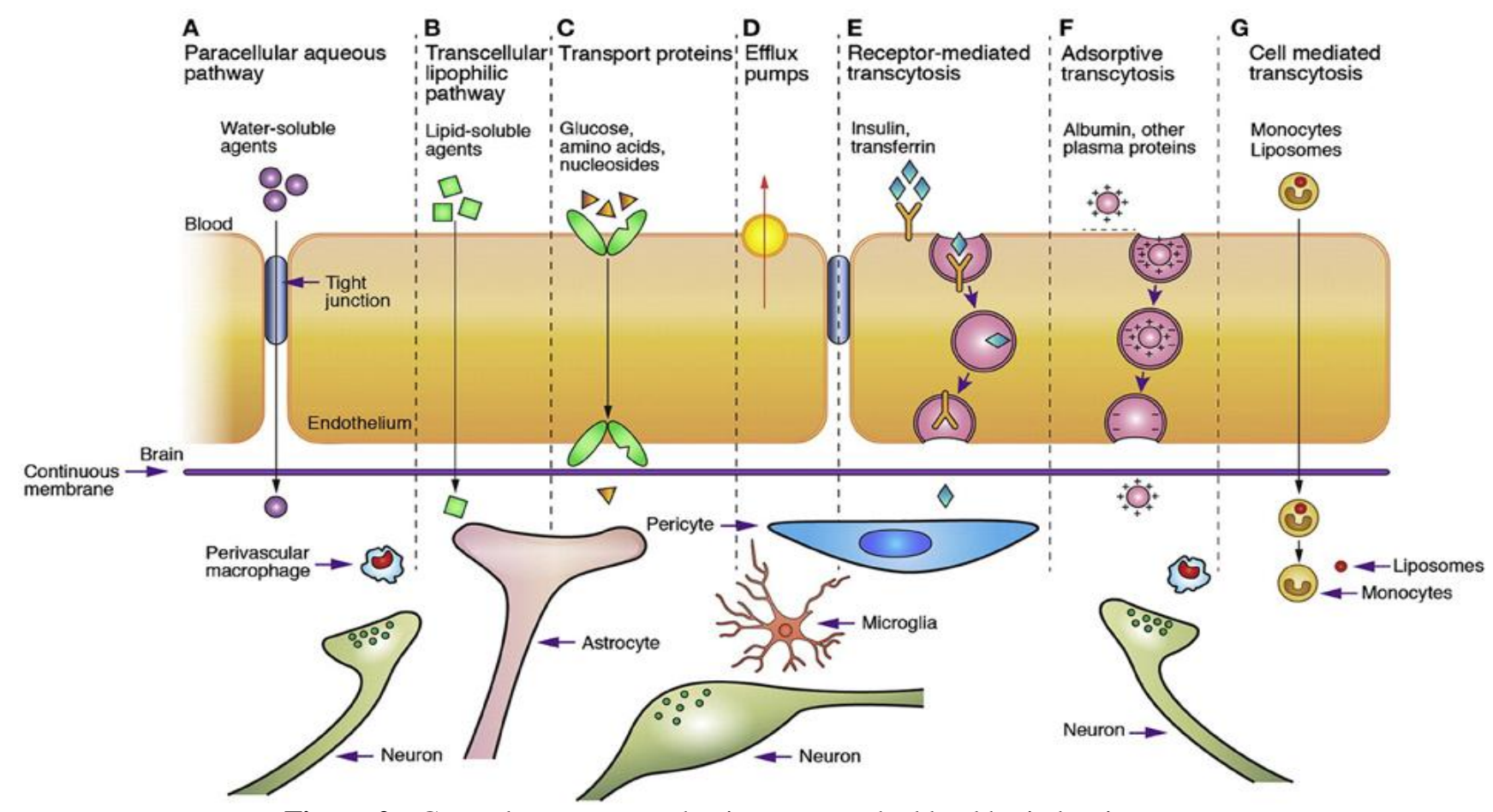

Figure 2:- General transport mechanisms across the blood brain barrier

\section{Nanomedicine:- Introduction:-}

Nanomedicine is defined as the application of nanotechnology to health. It exploits the improved and novel physical, chemical and biological properties of materials at the nanometric scale. Nanomedicine has potential impact on the prevention, early and reliable diagnosis and treatment of disease. It encompasses three interrelated themes of:

* Nanodiagnostics including imaging

* Targeted drug delivery and controlled release

* Regenerative medicine.

The ultimate goal of nanodiagnostics is to identify disease at the earliest stage possible. Nanotechnology can offer diagnostic tools of better sensitivity, specificity and reliability. The objective of drug delivery systems is to target selected cells or receptors within the body. This technique is driven by the need on one hand to more effectively target drugs to the site of disease, to increase patient acceptability and reduce healthcare costs; and on the other hand to deliver new classes of pharmaceuticals that cannot be effectively delivered by conventional means. The regenerative medicine is focused to work with the body's own repair mechanisms to prevent and treat disabling chronic disorders of cardiovascular and central nervous system. Rather than targeting the symptoms or attempting to delay the progress of disease, future therapies will be designed to rectify chronic conditions using body's own healing mechanisms. For example, promoting self-repair mechanisms in the areas of the central nervous system. The brain represents one of the most complex systems in biomedicine. With an improved understanding of brain functioning, nanotechnology offers better diagnosis and treatment for neurodegenerative disorders like multiple sclerosis, Alzheimer's disease and Parkinson's disease.

\section{Nanoscience as alzheimer's biomarker detector:-}

One of the most promising applications of nanoscience is in Alzheimer's disease. As patients can be definitely diagnosed after they pass way and their brain is examined for the telltale damage, scientists are hunting for tests that would help make a diagnosis in living patients. One possible biomarker for Alzheimer's is a protein called amyloid beta-derived diffusible ligands (ADDL). Support for the role of ADDLs comes from their neurotoxicity ${ }^{[37]}$, and presence at elevated levels in the brains of $\mathrm{AD}$ patients as compared with the age-matched controls ${ }^{[38}$. The correlation of CSF ADDL levels with disease state offers promise for improved AD diagnosis and early treatment. This finding was made possible by combining ADDL-specific monoclonal antibodies ${ }^{[9,40]}$ with an ultrasensitive, 
nanoparticle-based protein detection strategy termed biobarcode amplification (BCA) ${ }^{[41]}$. The BCA strategy used by Klein, Mirkin and coworkers ${ }^{[42,41]}$ makes clever use of nanoparticles as DNA carriers to enable millionfold improvements over ELISA sensitivity. CSF is first exposed to monoclonal anti-ADDL antibodies bound to magnetic microparticles. After ADDL binding, the microparticles are separated with a magnetic field and washed before addition of secondary antibodies bound to DNA:Au nanoparticle conjugates. These conjugates conatin covalently bound DNA as well as complementary "barcode" DNA that is attached via hybridization. Unreacted antibody:DNA:Au nanoparticle conjugates are removed during second magnetic separation, after which elevated temperature and low-salt conditions release the barcode DNA for analysis. Because the pathology of AD is thought to begin decades before the first symptoms, it would be very interesting to learn at what stage of disease progression ADDL levels in the CSF rise above those in healthy individuals.

\section{Nanotechnology based diagnosis of alzheimer's disease:-}

The recently growing application of nanotechnology in molecular detection of biomarkers is promising for very early diagnosis of Alzheimer's disease. From a practical point of view, one may perform a molecular detection process either inside the body (in vivo) or on the samples derived from the body (in vitro). Nanotechnology may help us to achieve early diagnosis of AD by providing us with a highly potent signal transduction approach. Signal transduction refers to the process through which a biological signal (a biomarker) transforms to a recordable signal, and is amplified enough to be recorded. This potential application of nanotechnology in molecular diagnosis is mainly based on the special physical (optical, electrical or magnetic), chemical and biological characteristics of certain multifunctional nanoparticles. In what follows we present and compare a number of methods, which have aimed to address this need.

Diagnostics and Imaging e.g. nanoparticle-based methods for early and sensitive detection of $A D$ biomarkers in CSF/blood; nanomaterials for in vivo imaging of beta-amyloid plaques (contrast agents for MRI, quantum dots,....)

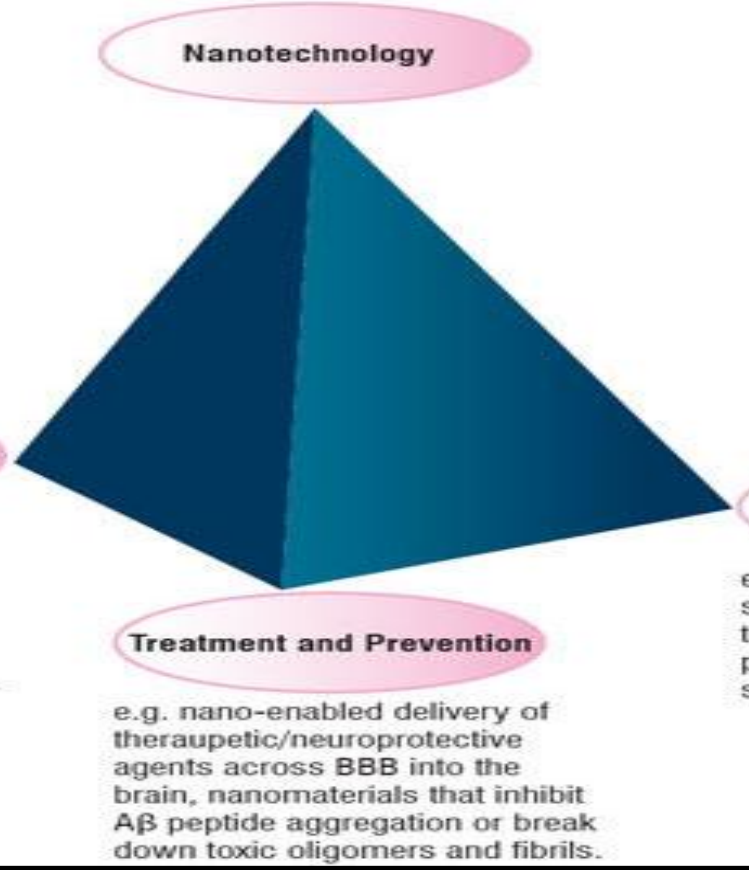

Regeneration Therapy e.g. nanomaterial scaffolds for on-site neural tissue regrowth, nanopatterned surfaces for stem-cell culturing.

\section{Ethical issues:-}

Figure 3:- Nanotechnology-Based Diagnosis of Alzheimer's Disease

Nanotechnology offers great promise for medicine, but much of this lies in future. This future orientation has made nanotechnology vulnerable to the current zeitgeist of over claiming in science, either the harm or benefit. There is a need to be careful about placing premature weight on speculative hopes or concerns about nanotechnologies ${ }^{4}$ raised ahead of evidence. Concern for economic competitiveness and other economic values may come into conflict with respect for human dignity. The unrestricted freedom of some may endanger the health and safety of others. Therefore a balance has to be struck between values that are all legitimate in our culture. Even though the technology is based on the tiniest of particles, the possible payoff is anything but small 


\section{Proposed in vitro nanodiagnostic approaches:-}

1. DNA Nanoparticles Conjugates (Bio-Barcode Assay): DNA Nanoparticle conjugates are capable of the attomolar scale detection of protein biomarkers ${ }^{17}$. Through a technique known as bio-barcode assay, ultra-low concentrations of protein biomarkers may be detected, owing to carrier gold nanoparticles that match the specific antibody of the target biomarker with hundreds of DNA barcodes. Therefore, a single molecule of biomarker may be traced by hundreds of DNA barcodes (a biological signal transformation), which could be additionally amplified by the polymerase chain reaction (PCR) technique ${ }^{18}$

2. Nanoparticle Surface Plasmon Resonance: Recently a method for the detection of molecular biomarkers was examined for $\mathrm{AD}$ biomarkers ${ }^{19}$ which is said to be ultra-sensitive and inexpensive. It is called the localized surface plasmon resonance (LSPR) nanosensor and it is based on singular optical properties of triangular silver nanoparticles (AgNPs).

3. Scanning Tunneling Microscopy System: Another recent development is a molecular detection system which was proposed based on electrical detection using a scanning tunneling microscope (STM) ${ }^{20,21}$. The settings of this technique included immobilization of specific antibody fragments on gold ( $\mathrm{Au}$ ) substrate and $\mathrm{Au}$ nanoparticles (AuNP).

4. Two-Photon-Rayleigh Spectroscopy: Recently, two-photon-Rayleigh scattering signal of AuNPs was examined as a transformed signal of an immunosensor for tau protein, one of $\mathrm{AD}$ biomarkers ${ }^{22 .}$ In this study, conjugates of AuNP with anti-tau antibody were used to detect tau proteins in a sample solution. The basis of biomarker signal transformation was the ensuing aggregation of AuNP-antibody conjugates after addition of tau protein. It is claimed that through this technique tau protein could be detected at concentrations as low as $1 \mathrm{pg} / \mathrm{ml}$ within 35 minutes. By using this technique, the investigators claimed to have introduced, for the first time, a fast, ultra-sensitive and specific nanosensor for detecting tau protein

\section{Proposed in vivo diagnostic approaches:-}

1. Micro Magnetic Resonance Imaging ( $\mu$ MRI): The usage of iron oxide nanoparticles as magnetic resonance imaging (MRI) contrast agents has been widely researched in the recent decades ${ }^{23,24}$. Two groups of investigators have reported the application of monocrystalline iron oxide nanoparticles (MION) and ultra-small superparamagnetic In sciences Journal | Nanotechnology ISSN 1664-171X iron oxide (USPIO) nanoparticles as MRI contrast agents for in vivo detection of amyloid peptide plaques in the brain of transgenic mouse model of $\mathrm{AD}^{25,26}$

2. Optical imaging: Another recently growing approach for in vivo detection of molecular biomarkers is optical imaging through special near-infrared (NIR) fluorescent contrast agents ${ }^{27}$. Due to the long wavelength, the scattered light from these contrast agents could penetrate through biological tissues. The common requirements for a molecular diagnostic probe for $\mathrm{AD}$ include the ability to cross the $\mathrm{BBB}$ and specifically target an $\mathrm{AD}$ related biomarker (e.g. A $\beta$ ).

3. Quantum dots: Quantum dots are another group of nanotechnology-made fluorescent dyes ${ }^{28,32}$. These nanoscale semiconductor crystals have special fluorescent properties including minimal photo bleaching, optimal stability, high signal to noise ratio and broad absorption spectrum with very narrow but size-dependant tunable emission spectrum. These advantages over conventional fluorescent dyes ${ }^{28}$ give QDs the potential for long-term tracking and simultaneous visualization of multiple physiological and pathological molecular events $^{29}$. This simultaneous multiple labeling property is especially important for diagnosis of AD, since there are several biomarkers in the AD pathology, and not only their existence but also their proportion to each other may be helpful for ruling out other differential diagnosis. 


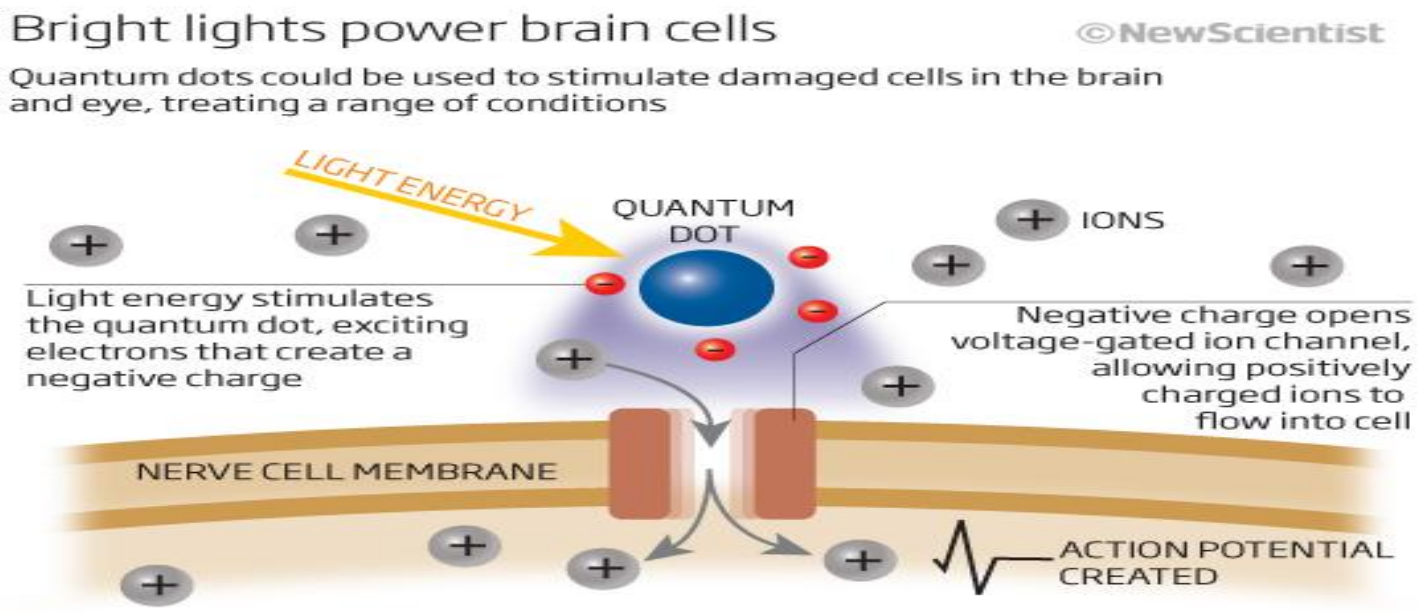

Figure 4:-Quantum dots stimulate damage cells in the brain.

Table 1:- Nanotechnology tools for alzheimers disease.

Currently available nanotechnology tools for $\mathrm{AD}$ diagnosis

\begin{tabular}{|c|c|c|c|c|}
\hline Nanotech tool & Nanoparticle & Biomarker & Detection mechanism & Signal transduction basis \\
\hline $\begin{array}{l}\text { BCA } \\
\text { (Bio-barcode assay) }\end{array}$ & Gold & ADDL & $\begin{array}{l}\text { Sandwich immunoassay } \\
\text { (monoclonal anti-ADDL } \\
\text { antibody): }\end{array}$ & $\begin{array}{l}\text { Simultaneous conjugation of Au NP } \\
\text { with the specific Antibody and numerous } \\
\text { DNA barcodes }\end{array}$ \\
\hline $\begin{array}{l}\text { LSPR } \\
\text { (Localized surface } \\
\text { plasmon resonance) }\end{array}$ & $\begin{array}{l}\text { Silver } \\
\text { (Triangular) }\end{array}$ & $\mathrm{ADDL}$ & $\begin{array}{l}\text { Sandwich immunoassay } \\
\text { (monoclonal \& polyclonal } \\
\text { anti-ADDL antibody): }\end{array}$ & $\begin{array}{l}\text { Sensitivity of silver nanoparticles } \\
\lambda \max \text { to their external nanoenvironment }\end{array}$ \\
\hline
\end{tabular}

$\mathrm{ADDL}=$ amyloid derived diffusible ligands; $\mathrm{NP}$. nanoparticle; $\lambda$ max: wavelength of maximum extinction.

\section{Alzheimer's disease therapy through nanotechnology:-}

Presently there exist no therapeutic methods available for curing AD. The cure forADwould require therapeutics that will cease the disease progress and willreverse its resultant damages. Today, common medications for AD are symptomatic and aim at the disrupted neurotransmission between the degenerated neurons. Examples of such medications are acetylcholine esterase inhibitors, including tacrine, donepezil, rivastigmine and galantamine .

With further research, mechanistic therapeutic approaches could gradually complement the above mentioned medications. Design of each mechanistic therapeutic is for targeting a different stage of the AD pathogenetic process and therefore help to cease the progress of the disease. Presently there are the following five molecular mechanistic therapeutic approaches under investigation

1. Inhibition of $\mathrm{A} \beta$ production;

2. Inhibition of $\mathrm{A} \beta$ oligomerization,

3. Anti-inflammation,

4. Cholesterol homeostasis modulating; and

5. Metal chelation.

Meanwhile, advances in nanotechnology are adding further opportunities for the AD therapy. Generally, the focus of the nanotechnology therapeutic approaches for every disease have been on drug discovery and monitoring , 
controlled release of therapeutic agents, and targeted drug delivery. The later is the most researched one, and it is especially prerequisite for reaching stronger therapeutic effects with the least amount of side effects. These applications are quite remarkable and challenging in respect to diseases of the CNS and brain. The subject of targeted drug delivery, for example, is appreciably complicated for CNS, due to the additional obstacle of the blood brain barrier (BBB) against the entry of a variety of molecules into the CNS tissues. With respect to drug discovery and monitoring, the histological complexity of CNS is a restricting factor. However, the potential capabilities of nanoparticles and nanodevices, including their controllable size and suspendability (based on modifiability of the Nanoparticles outer layer), multi-functionality and remote controlled functionality show promise in overcoming the CNS restrictions. Nevertheless, there are many challenges regarding the biocompatibility of Nanoparticles and nanodevices especially in a complex biological milieu like brain with a huge concentration of cells and intercellular communications.

\section{Nanotechnology based treatment for alzheimer's disease:-}

Nanomedicine engineered tunable devices with the size in the order of billionth of meters have been proposed as an intriguing tool potentially able to solve the unmet problem of enhancing drug transport across the BBB, currently available therapeutics for $\mathrm{AD}$, only act to lower its symptoms . In recent years, however, significant amount of research have been focused on finding the so called "neuroprotective agents", therapeutics that could stop the disease progress by targeting special molecular mechanisms in the AD pathology process ${ }^{30}$. However, more futuristic are approaches that could rebuild the damaged tissue, called as "regenerative agents". These two (neuroprotective and neuroregenerative) approaches together are known as "disease-modifying approaches". They are distinguished from symptomatic approaches by the fact that in addition to ameliorating the symptoms they are aimed to stop the disease progress and restore the dysfunctional or dead tissue ${ }^{30}$. The therapeutic potential of nanotechnology for $\mathrm{AD}$ includes both neuroprotective and neuroregenerative approaches. In addition, nanotechnology has shown promising applications in targeted drug delivery for $\mathrm{AD}$, and several nanocarrier systems have been studied in recent years to increase the bioavailability and efficacy of different AD therapeutic agents.

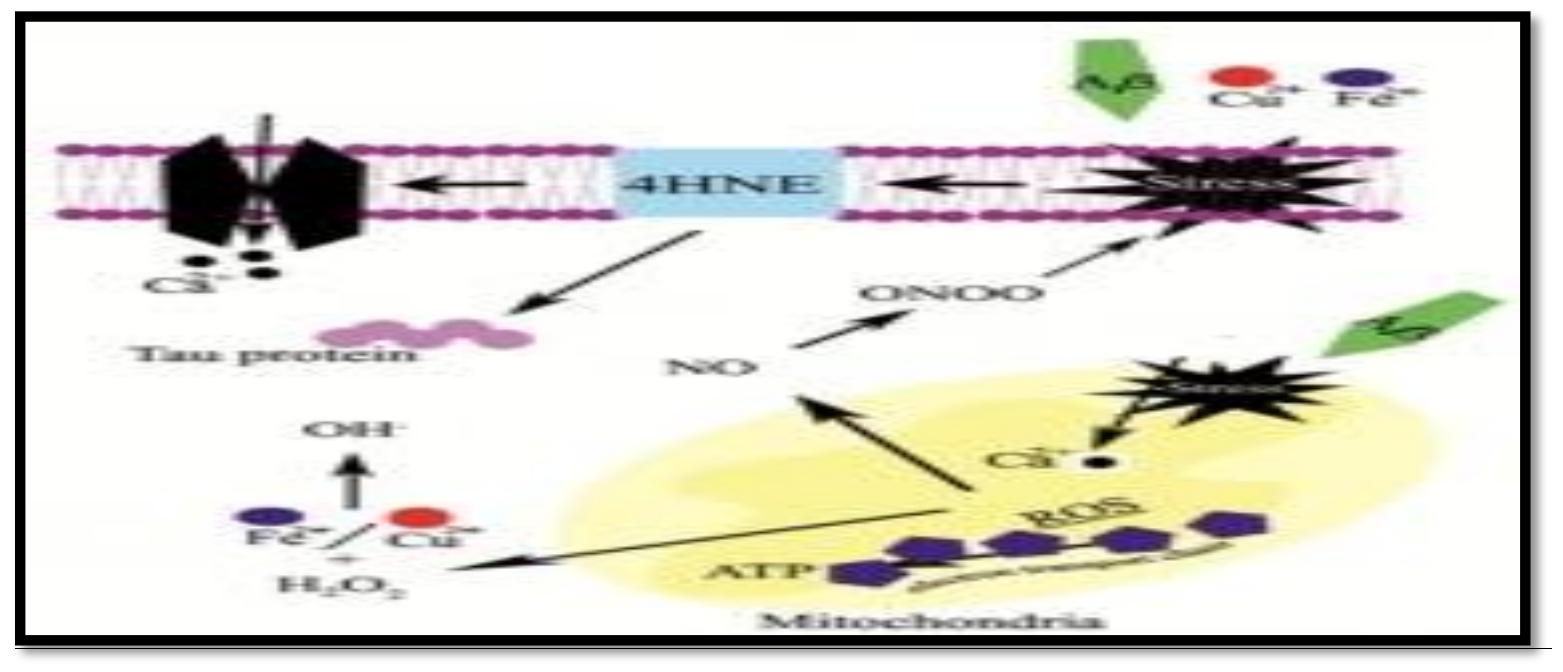

In the above figure 5 the interaction of $\mathrm{Fe}^{3+}$ (ferric) and $\mathrm{Cu}^{2+}$ (copper) with $\mathrm{A} \beta$ leading to the production of oxidative stress is shown. A $\beta$ can also oligomerize in the lipid bilayer of cell plasma membrane, leading to formation of membrane calcium channels ${ }^{[31]}$. These calcium channels cause an imbalance in calcium homeostasis, that ends in oxidative stress. In addition, the membrane integrated $\mathrm{A} \beta$ can chemically interact through (amino acid) Methionine (not shown) with the membrane lipid molecules and the resultant lipid peroxidation produces 4-hydroxy-2-nonenal $(4 \mathrm{HNE})^{[32]}$. Such an interaction leads to membrane disruption and production of reactive oxygen species and finally oxidative stress in the involved brain tissue. The 4HNE and other reactive oxygen species (ROS) also lead to tau phosphorylation and aggregation. Moreover, intracellular aggregates of $A \beta$ cause mitochondrial oxidative stress, and further imbalance in $\mathrm{Ca} 2+$ hemostasis. The resultant impairment of electron transfer chain leads to overproduction of superoxide anion, which is converted either to $\mathrm{H}_{2} \mathrm{O}_{2}$ or to peroxynitrite (following interaction with nitric oxide (NO)). The interaction of $\mathrm{H}_{2} \mathrm{O}_{2}$ with $\mathrm{Fe}^{2+}$ or $\mathrm{Cu}^{2+}$ produces the hydroxyl radical $\left(\mathrm{OH}^{*}\right)$, a strong $\mathrm{ROS}$ that induces membrane-associated oxidative stress ${ }^{30}$ 
Ligands and target genes of some nuclear receptors:-

Table 2:- Ligands and target genes of some nuclear receptors for Alzheimers Disease(AD)

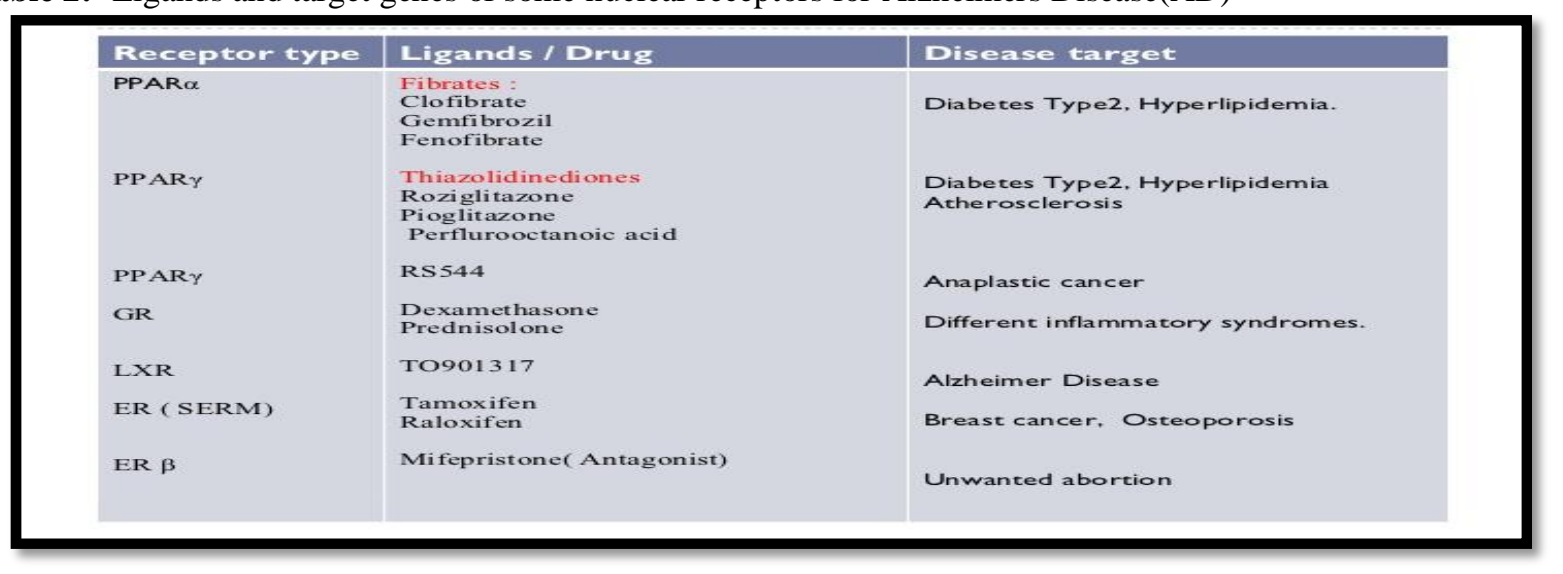

\section{Nanoformulations investigated:-}

Numerous nanoformulations have been investigated successfully for better brain delivery which includes nanoparticulate systems (polymeric/solid lipid), liposomes, dendrimers, nanoemulsions, nanosuspensions, and ligand mediated nanosystems.

1. Polymeric nanoparticles: Nanoparticles (NPs) are, colloidal particles, less than $1000 \mathrm{~nm}$, that can be used for better drug delivery and prepared either by encapsulating the drug within a vesicle and or by dispersing the drug molecules within a matrix ${ }^{32}$. Nanoparticulate drug delivery systems have been extensively studied in recent years for spatial and temporal delivery, especially in tumour and brain targeting. NPs have great promise for better drug delivery as found in both pharmaceutical and clinical research. As a drug carrier, NPs have significant advantages like better bioavailability, systemic stability, high drug loading, long blood circulation time and selective distribution in the organs/tissues with longer half life. Nanoparticles, prepared from a wide variety of biodegradable/biocompatible polymers such as poly(D,L-lactide-co-glycolide) (PLGA), poly(D,Llactide) (PLA), polybutyl cyanoacrylates (PBCA), polycaprolactone (PCL) etc are extensively used for the delivery of drugs to the central nervous system.

2. Solid lipid nanoparticles (SLN): Solid lipid nanoparticles (SLN) are colloidal particles composed of biocompatible/biodegradable lipid matrix that is solid at body temperature and exhibit size in a range of 100 to $400 \mathrm{~nm}^{33}$. SLN offer several advantages such as controlled drug release, targeted delivery, increased drug stability, high drug payload, least biotoxicity, large scale production and ease of sterilization. General ingredients used in the preparation of SLN are solid lipid(s), emulsifier(s) and water. The term "lipid" has a broader sense here and includes triglycerides (e.g. tristearin), fatty acids (e.g. stearic acid), partial glycerides (e.g. Imwitor), steroids (e.g. cholesterol) and waxes (e.g. cetyl palmitate). SLN are widely used for the delivery of active pharmaceutical ingredients to the brain because of the advantages mentioned above and its enhanced ability to cross BBB.

3. Liposomes: Nanoformulations such as liposomes consists of bilayer phospholipid systems in which watersoluble drugs could reside in the aqueous phase enveloped by phospholipid bilayer and the lipophilic drugs, could directly integrate into the membrane. Researchers are actively investigating on several advanced versions of liposomes such as long-circulating (PEGylated) liposomes, triggered release liposomes, liposomes containing nucleic acid polymers, ligand-targeted liposomes and liposomes containing combinations of drugs in order to achieve better drug delivery. Targeted brain delivery using liposomal systems resulted in considerable increase of drug concentration in brain/in vitro cell lines ${ }^{34}$.

4. Dendrimers: Dendrimers are a unique class of synthetic polymers which has a major role in nanotechnological advances of drug delivery. The term "dendra" in "dendrimer" is derived from Greek which means tree and therefore appropriately describes its architecture. Novel dendrimer-based drug delivery systems consisting of G3 polyamidoamine (PAMAM) and surfactant conjugated dendritic nanoconjugates have been successfully applied for targeted brain delivery ${ }^{35}$. Cytotoxicity studies showed that free G3 PAMAM was relatively nontoxic while the conjugation of lauryl chains and paclitaxel molecule on the surface of G3 PAMAM dendrimer significantly increased the cytotoxicity in both human colon adenocarcinoma cell line (Caco-2) and primary cultured porcine brain endothelial cells (PBECs). Enhanced permeation of the lauryl-modified G3 PAMAM dendrimer-paclitaxel conjugates across Caco-2 cell and PBEC monolayers has also been demonstrated. 
Dendrimer conjugate had approximately 12 -fold greater permeability across both cell monolayers than that of paclitaxel alone.

5. Micelles: Polymeric micelles obtained from block copolymers as colloidal carriers for drug and gene targeting have been receiving much attention in the field of drug delivery and targeting because of the high drug-loading capacity. A variety of drugs with diverse characteristics, including genes and proteins, can be incorporated into the core. Researchers have demonstrated effective targeting of micelles systems to the brain by intra venous as well as intra nasal route ${ }^{36}$.

6. Nanoemulsions: Nanoemulsions have also gained considerable attention in research as well as in therapeutics due to their advantages such as ease of preparation, thermodynamic stability, optical clarity, and their ability to incorporate both hydrophobic and hydrophilic solutes etc. Intranasal nanoemulsion based brain targeting drug delivery system of risperidone was studied by Mukesh Kumar et al. They have found higher drug transport efficiency (DTE\%) and direct nose to brain drug transport (direct transport percentage, DTP\%) for these mucoadhesive nanoemulsions. Similar results have been obtained with saquinavir-loaded nanoemulsions which 40 also resulted in efficient brain delivery ${ }^{37}$.

7. Ligand-Mediated Active Targeting: Advances in cell biology with respect to internalization pathways and problems associated with delivery of new macromolecular drugs such as peptides and proteins paved the path of receptor mediated targeting for selective uptake and internalization of drugs. The flexibility of nanoformulations for the attachment of ligands for specific receptors further improved the scope of ligand mediated active targeting since these systems also provide additional advantages such as controlled release of drugs and protection from external degradation before reaching the targeted site. Numerous receptors which are over expressed in brain such as transferrin receptors, insulin receptors, low density lipoprotein receptors etc have been widely explored for the ligand mediated targeted brain delivery.

8. Nanogels: Nanogels designed an amphipathic nanogel that incorporates proteins and controls their folding and aggregation, similar to natural chaperones (proteins assisting the non-covalent folding and/or unfolding). In the case of $\mathrm{A} \beta$, these nanogels would inhibit the amyloidogenesis process effectively through this mechanism The nanogel (hydrogel nanoparticles) designed in this study was composed of cholesterol bearing pullulan (CHP). Pullulan is a natural water-soluble polysaccharide polymer consisting of maltotriose (a trisaccharide consisting of three glucose molecules linked with 1,4 glycosidic bonds) units. Inhibiting assembly at the monomer level, this technique prevents $A \beta$ oligomerization and therefore reduces the concentration of toxic $A \beta$ oligomeric species. Recently, Boridy et al. demonstrated a significant reduction in A $\beta 42$ toxicity in the primary cortical cell culture and microglial cell culture after using CHP nanogels ${ }^{38}$

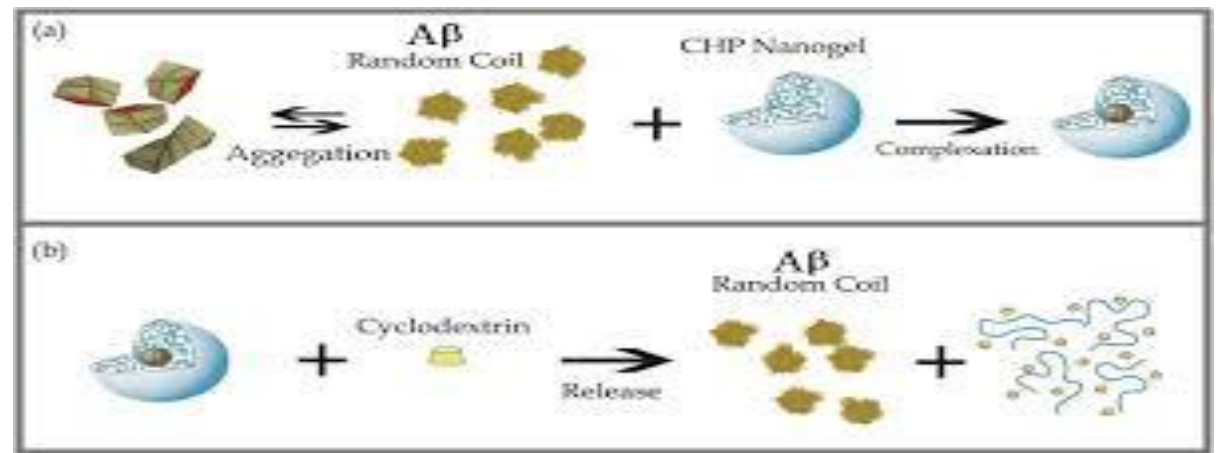

Figure 6:- (a). Schematic representation of the interactions between artificial nanoscale chaperone system and misfolded $A \beta$. (b). Refolded $A \beta$ monomers are released after addition of cyclodextrin.

9. Fullerene: Fullerene $\left(\mathrm{C}_{60}\right)$ and its derivatives could be the base of neuroprotective compounds . The biological applications of fullerene, including its anti-oxidant and free radical scavenger potentials, are due to its kind of chemical structure that allows it to be linked (and to be functionalized) by several active chemical groups in a 3dimensional orientation. ${ }^{39}$

10. Nano-Ceria: Cerium oxide $\left(\mathrm{CeO}_{2}\right)$ nanoparticle (nano-ceria) is reported to have neuroprotective effects on $\mathrm{AD}$ in vitro models. This is mainly due to the anti-oxidant properties of nano-ceria, originating in the two oxidation-reduction (redox) states of cerium: $\mathrm{Ce}^{2+}$ and $\mathrm{Ce}^{4+}$ and the resultant oxygen vacancies. D'Angelo et al. showed that in addition to the mentioned anti-oxidant properties, nano-ceria protects neurons from cytotoxic

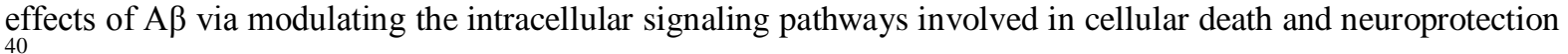




\section{Conclusion:-}

Alzheimers Disease(AD) is the primary common dementia syndrome worldwide, with an astronomical devastating socioeconomic burden on society. Solving the major problems of early diagnosis and effective cure for AD requires interdisciplinary research efforts. Early diagnosis need to be performed before the underlying pathology has become severe enough to present itself clinically. Several molecular biomarkers are recognized to be associated with the pathology of $\mathrm{AD}^{37}$. Research on the basic pathogenetic mechanisms of the disease has provided new insight for designing diagnostic and therapeutic methods. However, the need for further studies on the exact root causes of AD is still palpable

\section{Applications of nanotechnology methods in studies on the molecular mechanism of AD are promising:-}

1. Through Atomic force microscopy(AFM).studies on a number of direct in vitro observations of the main pathological structures of Alzheimers Disease(AD), A $\beta$ species and $\tau$-protein are accomplished. Using the AFM tapping mode operation and applying multi-wall carbon nanotubes as the AFM tips has enabled high-resolution visualization of $\mathrm{A} \beta$ and $\tau$ during their related chemical processes. The results illuminate the mechanisms of these key processes in $\mathrm{AD}$ and the structural properties of their involved molecules.

2. Through single-molecule FRET (fluorescence resonance energy transfer), which is applicable both in vivo and in vitro, studies into the submolecular and intermolecular interactions of abnormal protein species in $\mathrm{AD}$ pathogenetic process has been achieved.

3. In addition, using NanoSIMS microscopy has enabled investigation into subcellular chemical elemental changes during the $\mathrm{AD}$ pathogenesis . Nanotechnology offers a number of highly sensitive molecular detection tools that may make this goal achievable. These nanodiagnostic tools utilize different nanoparticles/nanostructures, and are based on different physicochemical interactions that may be utilized either in vitro or in vivo. Alzheimer's disease seems to be a multi-factorial disease with several pathogenetic mechanisms and pathways, which are not necessarily interrelated. Therefore, it would be a great step forward for Alzheimer's disease therapy if a multifunctional nanotechnology approach could be developed for designing therapeutic cocktails that simultaneously and specifically target the main molecular culprits involved in AD. These molecular targets include (but are not limited to) $\mathrm{A} \beta$ oligomers, Reactive oxygen species(ROS), excessive metal ions, tau phosphorylating kinases and cell cycle proteins. Nevertheless, there are still many challenges regarding the biocompatibility of nanoparticles and nanodevices especially in a complex biological milieu like brain with a huge concentration of cells. Therefore, it seems that a long and puzzling path is ahead to make the envisioned nano-neurosurgical approaches of curing CNS diseases a practical technology and eventually a routine clinical practice.

\section{Summary of applications of nanotechnology in treatment of alzhiemers disease:-}

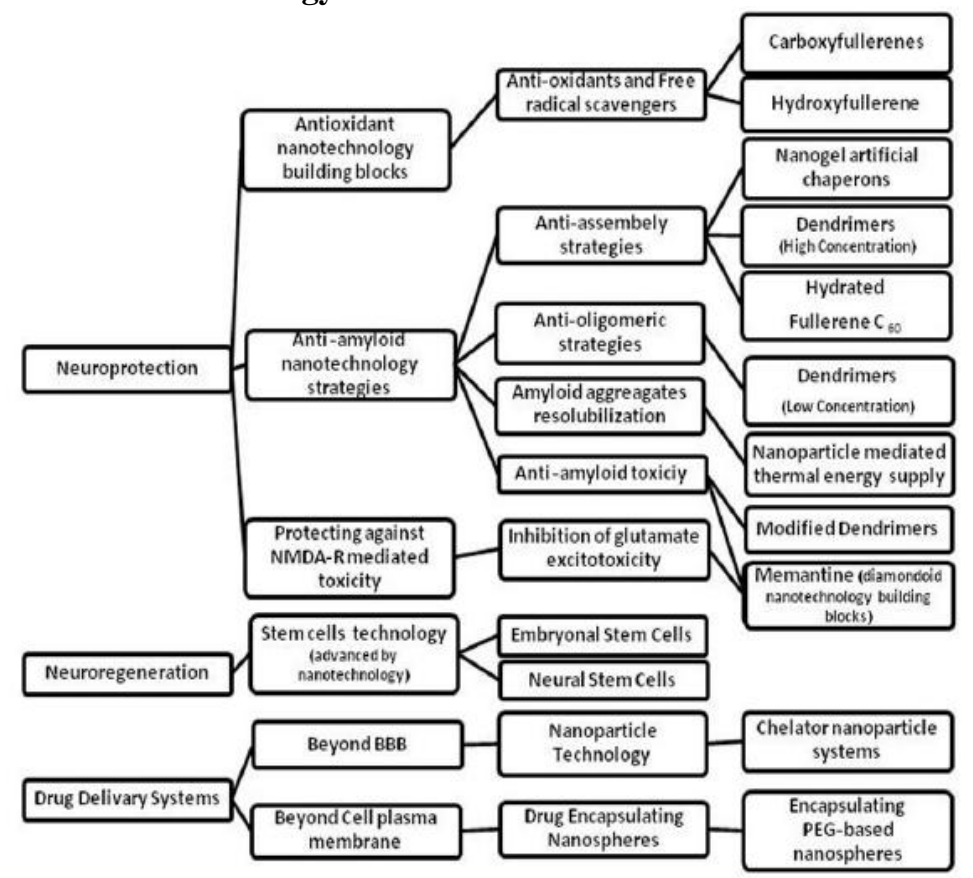




\section{References:-}

1. Azem A, Mansoori GA. Nanotechnology solutions for Alzheimer's disease: advances in research tools, diagnostic methods and therapeutic agents. J Alzheimers Dis 2008;Pg no:-199-223.

2. Alzheimer A. Ueber eine eigenartige Erkrankung der Himrinde. Allg Z Psychiat Med 2007Pg no:-146-148.

3. Ferri CP, Prince M, Brayne C, Brodaty H, Fratiglioni L, Ganguli M, et al. Global prevalence of dementia: a Delphi consensus study. Lancet 2005 Dec 17; 366(9503):Pg no:-2112-2117.

4. Sloane PD, Zimmerman S, Suchindran C, Reed P, Wang L, Boustani M, et al. The public health impact of Alzheimer's disease, 2000-2050: potential implication of treatment advances. Annu Rev Public Health 2002; 23:Pg no:-213-231.

5. Mortimer JA, Borenstein AR, Gosche KM, Snowdon DA. Very early detection of Alzheimer neuropathology and the role of brain reserve in modifying its clinical expression. J Geriatr Psychiatry Neurol 2005 Dec; Pg no:218-23.

6. Nestor PJ, Scheltens P, Hodges JR. Advances in the early detection of Alzheimer's disease. Nat Med 2004 Jul; 10 Suppl:S34-S41.

7. Mucke L. Neuroscience: Alzheimer's Disease. Nature 2009; Pg no:895-897.

8. Murman DL, Colenda CC. The economic impact of neuropsychiatric symptoms in Alzheimer's disease: can drugs ease the burden? Pharmacoeconomics 2005; 23(3)Pg no:227-232.

9. Uversky VN, Kabanov AV, Lyubchenko YL. Nanotools for megaproblems: probing protein misfolding diseases using nanomedicine modus operandi. J Proteome Res 2006 Oct; 5(10):2505- 22.

10. Mansoori GA, Mohazzabi P, McCormack P, Jabbari S. Nanotechnology in cancer prevention, detection and treatment: bright future lies ahead. WRSTSD 2007; 4(2/3):Pg no:226-257.

11. Singh S, Singh M, Gambhir IS. Nanotechnology for Alzheimer's disease detection. Digest Journal of Nanomaterials and Biostructures 2008; 3(2):Pg no75-79.

12. Mansoori GA. Principles of Nanotechnology: Molecular-Based Study of Condensed Matter in Small Systems. World Scientific Pub. Co.; 2005.

13. Jain KK. The role of nanobiotechnology in drug discovery. Drug Discov Today 2005 Nov 1; 10(21): Pg no:1435-1442.

14. Cardoso FL, Brites D, Brito MA. Looking at the blood-brain barrier: Molecular anatomy and possible investigation approaches. 2010; 64:Pg no:-328-363.

15. Weiss N, Miller F, Cazaubon S, Couraud PO. The blood-brain barrier in brain homeostasis and neurological diseases. Biochim Biophys Acta Biomembr 2009; 1788: Pg no:-842-857.

16. 16.Chen Y, Liu L. Modern methods for delivery of drugs across the blood-brain barrier. Adv Drug Del Rev 2012; 64:Pg no:-640-665.

17. Georganopoulou DG, Chang L, Nam JM, Thaxton CS, Mufson EJ, Klein WL, et al. Nanoparticlebased detection in cerebral spinal fluid of a soluble pathogenic biomarker for Alzheimer's disease. Proc Natl Acad Sci U S A 2005; 102(7):Pg no:- 2273-2276.

18. Haes AJ, Chang L, Klein WL, Van Duyne RP. Detection of a biomarker for Alzheimer's disease from synthetic and clinical samples using a nanoscale optical biosensor. J Am Chem Soc 2005; 127(7): Pg no:-2264-2271.

19. Kang DY, Lee JH, Oh BK, Choi JW. Ultra-sensitive immunosensor for -amyloid (1-42) using scanning tunneling microscopy-based electrical detection. Biosens Bioelectron 2009; 24(5):Pg no:-1431-1436.

20. Neely A, Perry C, Varisli B, Singh AK, Arbneshi T, Senapati D, et al. Ultrasensitive and highly selective detection of Alzheimer's disease biomarker using two-photon Rayleigh scattering properties of gold nanoparticle. ACS Nano 2009; 3(9) Pg no:2834-2840.

21. Skaat H, Margel S. Synthesis of fluorescent-maghemite nanoparticles as multimodal imaging agents for amyloid-beta fibrils detection and removal by a magnetic field. Biochem Biophys Res Commun 2009; 386(4): Pg no:-645-649.

22. Hofmann-Amtenbrink M, Hofmann H, Montet X. Superparamagnetic nanoparticles - a tool for early diagnostics. Swiss Med Wkly 2010; 140(w13081).

23. Wadghiri YZ, Sigurdsson EM, Sadowski M, Elliott JI, Li Y, Scholtzova H, et al. Detection of Alzheimer's amyloid in transgenic mice using magnetic resonance microimaging. Magnetic Resonance in Medicine 2003; 50(2): Pg no:-293-302.

24. Yang J, Wadghiri YZ, Hoang DM, Tsui W, Sun Y, Chung E, et al. Detection of amyloid plaques targeted by USPIO-A-beta 1-42 in Alzheimer's disease transgenic mice using magnetic resonance microimaging. NeuroImage 2011; 55, Pg no:-1600-1609. 
25. Nesterov EE, Skoch J, Hyman BT, Klunk WE, Bacskai BJ, Swager TM. In Vivo Optical Imaging of Amyloid Aggregates in Brain: Design of Fluorescent Markers. Angew Chem Int Ed Engl 2005; 44[34], Pg no:- 54525456.

26. Willets KA, Ostroverkhova O, He M, Twieg RJ, Moerner WE. Novel fluorophores for singlemolecule imaging. J Am Chem Soc 2003; 125[5], Pg no:-1174-1175.

27. Jain KK. Nanotechnology in clinical laboratory diagnostics. Clin Chim Acta 2005; 358[1-2], Pg no:-37- 54

28. Cummings JL. Defining and labeling disease-modifying treatments for Alzheimer's disease. Alzheimer's \& Dementia 2009; 5:Pg no:406-418.

29. Quist A, Doudevski I, Lin H, Azimova R, Ng D, Frangione B, et al. Amyloid ion channels: a common structural link for protein-misfolding disease. Proc Natl Acad Sci U S A 2005 Jul 26; 102(30):10427-32. Butterfield DA, Boyd-Kimball D. The critical role of methionine 35 in Alzheimer's amyloid ?- peptide (1-42)-induced oxidative stress and neurotoxicity. Biochim Biophys Acta Proteins Proteomics 2005; 1703(2):Pgno:-149-156.

30. Ramos CP, Agulla J, Argibay B, Pérez MM, Castillo J. Serial MRI study of the enhanced therapeutic effects of liposomeencapsulated citicoline in cerebral ischemia. Int J Pharm 2011; 405:Pg no:-228-233.

31. Joshi MD, Müller RH. Lipid nanoparticles for parenteral delivery of actives. Eur J Pharm Biopharm 2009; 71:Pg no:-161-172.

32. Bellavance MA, Poirier MB, Fortin D. Uptake and intracellular release kinetics of liposome formulations in glioma cells. Int J Pharm 2010; 395:Pg no:-251-259.

33. Gajbhiye V, Jain NK. The treatment of Glioblastoma Xenografts by surfactant conjugated dendritic nanoconjugates. Biomater 2011; 32:Pg no:-6213-6225

34. Kataoka K, Harada A, Nagasaki Y. Block copolymer micelles for drug delivery: Design, characterization and biological significance. Adv Drug Del Rev 2012; 64: Pg no:-37-48.

35. Abdelbary GA, Tadros MI. Brain targeting of olanzapine via intranasal delivery of core-shell difunctional block copolymer mixed nanomicellar carriers: In vitro characterization, ex vivo estimation of nasal toxicity and in vivo biodistribution studies. Int J Pharm 2013; 452:Pg no:-300-310

36. Boridy S, Takahashi H, Akiyoshi K, Maysinger D. The binding of pullulan modified cholesteryl nanogels to Ab oligomers and their suppression of cytotoxicity. Biomaterials 2009; 30[29], Pg no:-5583- 5591.

37. Dugan LL, Lovett EG, Quick KL, Lotharius J, Lin TT, O'Malley KL. Fullerene-based Antioxidants and neurodegenerative disorders. Parkinsonism Relat Disord 2001 Jul; 7(3):Pg no:-243-246.

38. D'Angelo B, Santucci S, Benedetti E, Di Loreto S, Phani RA, Falone S, et al. Cerium oxide nanoparticles trigger neuronal survival in a human Alzheimer disease model by modulating BDNF pathway. Curr Nanosci 2009; 5(2):Pg no:-167-176. 